\title{
DRD4 48 bp multiallelic variants as age-population-specific biomarkers in attention-deficit/hyperactivity disorder
}

Cristian Bonvicini $\mathbb{1}^{1}$, Samuele Cortese $\mathbb{E}^{2,3,4,5,6}$, Carlo Maj $\mathbb{C}^{7}$, Bernhard T. Baune $\mathbb{B}^{8,9,10}$, Stephen V. Faraone $\mathbb{D}^{11,12}$ and Catia Scassellati $\mathbb{1}^{13}$

\begin{abstract}
The identification of biomarkers to support the diagnosis and prediction of treatment response for attention-deficit/ hyperactivity disorder (ADHD) is still a challenge. Our previous works highlighted the DRD4 (dopamine receptor D4) as the best potential genetic marker for childhood diagnosis and methylphenidate (MPH) response. Here, we aimed to provide additional evidence on biomarkers for ADHD diagnosis and treatment response, by using more specific approaches such as meta-analytic and bioinformatics tools. Via meta-analytic approaches including over 3000 cases and 16,000 controls, we demonstrated that, among the different variants studied in DRD4 gene, the 48-base pair, Variable Tandem Repeat Polymorphism, VNTR in exon 3 showed an age/population-specificity and an allelic heterogeneity. In particular, the 7R/"long" allele was identified as an ADHD risk factor in European-Caucasian populations $(d=1.31,95 \% \mathrm{Cl}: 1.17-1.47, Z=4.70 / d=1.36,95 \% \mathrm{Cl}: 1.20-1.55, Z=4.78$, respectively), also, from the results of last meta-analysis, linked to the poor MPH efficacy. The 4R/"short" allele was a protective factor in EuropeanCaucasian and South American populations ( $d=0.83,95 \% \mathrm{Cl}: 0.75-0.92, Z=3.58$ ), and was also associated to positive $\mathrm{MPH}$ response. These results refer to children with ADHD. No evidence of such associations was detected for adults with persistent ADHD (data from the last meta-analysis). Moreover, we found evidence that the $4 R$ allele leads to higher receptor expression and increased sensitivity to dopamine, as compared with the $7 \mathrm{R}$ allele $(d=1.20,95 \% \mathrm{Cl}$ : $0.71-1.69, Z=4.81$ ), and this is consistent with the ADHD protection/susceptibility effects of the respective alleles. Using bioinformatics tools, based on the latest genome-wide association (GWAS) meta-analysis of the Psychiatry Genomic Consortium (PGC), we demonstrated that the 48 bp VNTR is not in Linkage Disequilibrium with the DRD4 SNPs (Single Nucleotide Polymorphisms), which were not found to be associated with ADHD. Moreover, a DRD4 expression downregulation was found in ADHD specific brain regions (Putamen, $Z$ score $=-3.02, P=0.00252$ ). Overall, our results suggest that DRD4 $48 \mathrm{bp}$ VNTR variants should be considered as biomarkers to support the diagnosis of ADHD and to predict MPH response, although the accuracy of such a biomarker remains to be further elucidated.
\end{abstract}

\section{Introduction}

Attention-deficit/hyperactivity disorder (ADHD) is a complex neurodevelopmental disorder, characterized by

\footnotetext{
Correspondence: Catia Scassellati (c.scassellati@fatebenefratelli.eu)

${ }^{1}$ Molecular Markers Laboratory, IRCCS Istituto Centro San Giovanni di Dio

Fatebenefratelli, Brescia, Italy

${ }^{2}$ Center for Innovation in Mental Health, Academic Unit of Psychology،

University of Southampton, Southampton SO17 1BJ, UK

Full list of author information is available at the end of the article.

These authors contributed equally: Stephen V. Faraone, Catia Scassellati
}

age-inappropriate symptoms of inattention and/or hyperactivity-impulsivity, with a heterogeneous clinical phenotype ${ }^{1}$. The worldwide prevalence among school-aged children is around $5 \%^{2}$. About $65 \%$ of affected individuals continue to exhibit impairing ADHD symptoms into adulthood $^{3}$. ADHD prevalence in adults is estimated at $2.5 \%$.

The severity level and presentation of ADHD changes over the lifespan, with adult patients displaying less obvious symptoms of hyperactivity and impulsivity ${ }^{5}$. 
Moreover, changes in structural brain abnormalities from childhood to adulthood with ADHD have been reported ${ }^{6}$, suggesting potential differential causes for the onset and persistence of the disorder ${ }^{7}$.

ADHD aetiology is not yet completely understood. Despite evidence that environmental factors (e.g., maternal smoking, low birth weight, and prematurity) play a significant role, genetic studies support a strong genetic contribution. Indeed, average heritability was estimated at $76 \%^{8,9}$, in childhood and at $30-50 \%^{10-12}$ or even greater $^{13,14}$ in adulthood. The most recent and largest genome-wide association (GWAS) meta-analysis from the Psychiatric Genomics Consortium (PGC) identified common single-nucleotide (SNPs) variants, surpassing genome-wide significance in 12 independent loci ${ }^{15}$, providing important new insights into the neurobiology of childhood ADHD. Additional insight comes from the studies on the crucial role played by rare variants ${ }^{9}$.

Pharmacotherapy is a crucial component for the treatment of ADHD ${ }^{16}$. Taking into account both efficacy and safety, evidence from a recent network meta-analysis ${ }^{17}$ supports methylphenidate (MPH) in children and adolescents, and amphetamines, in adults, as possible firstchoice medications for the short-term treatment of ADHD, suggesting once again potential neurobiological differences across the lifespan.

In the era of precision medicine, the biomarker approach to diagnosis and treatment offers the opportunity to improve diagnostic assessment and provides insights into etiological mechanisms. As it is known that a considerable proportion (35\%) of ADHD patients do not respond to available first line medication, this approach has also the potential to contribute to individualized therapies. The DRD4 (dopamine receptor D4) is a G-protein-coupled receptor belonging to the D2-like receptor family, which modulates intracellular signalling by inhibiting the production of the second messenger cyclic AMP (cAMP) level ${ }^{18,19}$ and is responsible for neuronal signalling in the mesolimbic system of the brain. It is specifically involved in dopamine synthesis, release and neuronal firing ${ }^{18}$. It has been considered a candidate for the aetiology of ADHD due to its high expression in brain regions implicated in attention and inhibition, such as the orbitofrontal and anterior cingulate corte $\mathrm{x}^{20,21}$. Additional interest derived from a link with the personality trait of novelty seeking $^{22,23}$, which has been compared with the high levels of impulsivity and excitability often seen in $\mathrm{ADHD}^{24}$. Further, the DRD4 "knockout" mouse exhibits a heightened response to cocaine and methamphetamine relative to controls, as indicated by increases in loco-motor behaviour $^{25}$. The DRD4 gene comprises four exons and encodes a putative 387-amino acid protein with seven transmembrane domains, where the most widely studied $48 \mathrm{bp}$ VNTR (variable tandem repeat) polymorphism encodes the third cytoplasmic loop. This multiallelic polymorphism includes 11 copies of a 48-bp repeat sequence, where the 4, 7 and 2 repeat (R) alleles are the most prevalent. Genetic demographic studies report that the $7 R$ allele is present in highly varying percentages in different populations worldwide ${ }^{26-30}$. It is known that this polymorphism impacts on mRNA and protein expression levels, indicating a significant functional biological effect of this polymorphism on the translation of the respective protein $^{31}$. After the exon 3 VNTR, the other DRD4 polymorphisms studied are found in the promoter region of the gene: $120 \mathrm{bp}$ duplication (rs4646984); $-521 \mathrm{C} / \mathrm{T}$ (rs1800955), -616 C/G (rs747302); 12 bp (rs4646983), $-615 \mathrm{~A} / \mathrm{G}$ (rs936462), $-376 \mathrm{C} / \mathrm{T}$ (rs916455).

In our previous works ${ }^{7,32,33}$, we strongly suggested that DRD4 along with dopamine transporter gene (SLC6A3) are significant predictors of childhood ADHD susceptibility, different endophenotypes, MPH response, and linked to altered genes expression levels. However, the latest GWAS/meta-analysis ${ }^{15}$ did not detect associations with these "classical" candidate genes.

Here, we build on and expand our previous studies, focusing on $D R D 4$, to further assess its role as a potential biomarker for the diagnosis of ADHD and for MPH response, both in children and adults. Up-date and new meta-analyses were performed to statistically assess the association with ADHD in childhood and to confirm the functional role of the $48 \mathrm{bp}$ VNTR. Bioinformatics in silico analyses were conducted to understand the impact of DRD4 gene and of $48 \mathrm{bp}$ VNTR polymorphism in the pathology and to reconcile our positive findings with the negative results for five DRD4 SNPs in the GWAS of Demontis et al. ${ }^{15}$. We used also bioinformatics tools to confirm the functional role of DRD4 in specific ADHD brain regions. In addition, after the literature research on the association between DRD4 polymorphisms and ADHD susceptibility in children with ADHD and MPH response in ADHD adulthood, we concluded that there are not enough studies to perform meta-analyses.

So far as the literature research does not add further studies to the meta-analytic approach, we reported the results from the last more recent meta-analyses, and this regards the associations of SNPs and ADHD susceptibility in children with ADHD, as well as the $48 \mathrm{bp} / \mathrm{SNPs}$ with ADHD susceptibility in adulthood and with MPH response in ADHD childhood and adulthood.

\section{Materials and methods \\ Meta-analysis \\ DRD4 polymorphisms in children with ADHD}

Search strategy and selection criteria According to the PRISMA guidelines ${ }^{34}$, we searched the electronic databases PubMed, Embase and "ADHDgene Database" (http://adhd. psych.ac.cn/), up to December 2018, with no restrictions 
on language, date, or article type. In PubMed, we used the following search terms/syntax "ADHD OR attention deficit OR attention-deficit OR attention deficit hyperactivity disorder OR attention-deficit hyperactivity disorder OR hyperkinetic syndrome OR hyperkinetic disorder OR hyperactivity disorder OR hyperactive child syndrome" AND "children OR child" AND "DRD4 OR dopamine receptor D4, AND "gene", AND "polymorphisms", AND "SNP OR Single Nucleotide polymorphism", AND "VNTR OR variable tandem repeats", AND "association", AND "TDT OR Transmission Disequilibrium Test, OR familybased" AND "methylphenidate OR MPH", AND "pharmacogenetics", AND "drugs", AND "treatments", AND "clinical trials" AND "meta-analy" OR metaanaly". During the research, we identified different meta-analyses, however we took in consideration those more recent: Gizer and colleagues $^{35}$, Wu and colleagues ${ }^{36}$; Nikolaidis and Gray ${ }^{37}$; Myer and colleagues ${ }^{38}$, to cross-check their references to find any publications possibly missed in our electronic search. The literature search was performed independently by two individuals (CS, CB). Disagreements were resolved by the other authors.

The Newcastle-Ottawa Scale was used to assess quality of studies ${ }^{39}$.

Inclusion and exclusion criteria We selected articles that met the following inclusion criteria: ADHD diagnosis according to the Diagnostic and Statistical Manual of Mental Disorders (DSM-III, DSM-III-R, DSM-IV, DSMIV-TR) or equivalent Hyperkinetic disorder or the International Classification of Diseases 10th Revision (ICD-10) or previous versions; case-control and a family-based study design for genetic studies; clinical trials for pharmacogenetic studies. We excluded studies (a) using comparisons with a family control (healthy siblings, to avoid the deviation from Hardy-Weinberg Equilibrium); (b) using samples fully overlapping with other included studies; (c) for which data to perform analyses were not available, even after contacting the study corresponding authors.

Data extraction for meta-analyses CS and CB independently extracted the following data: first author, study design, year of publication, populations studied, study design, sample size, ethnic groups, and key results from each study.

Statistical analyses Review Manager was used to perform the meta-analysis (RevMan Version 5.1.6; Copenhagen, The Nordic Cochrane Centre, The Cochrane Collaboration, 2008). We used the random-effects model to generate a pooled effect size and $95 \%$ confidence interval (CI) from individual study effect sizes (the odd ratios for genetics studies using the Mantel-Haenszel,
$\mathrm{M}-\mathrm{H})$. The significance of the pooled effect sizes was determined by $z$-tests. Between-study heterogeneity was assessed using a $X^{2}$ test of goodness of fit test and the $I^{2}$ statistic. We used a $P$-value $<0.05$ to indicate statistical significance.

Publication bias was estimated using the method by Egger and colleagues ${ }^{40}$ which relies on a linear regression approach to measure funnel plot asymmetry on the natural logarithm scale of the effect size. The significance of the intercept (a) was determined by the $t$-test ${ }^{40}$. The rank correlation method and regression method tests were conducted using MIX version 1.7 (http://www.mixfor-meta-analysis.info).

In relation to $48 \mathrm{bp}$ multiallelic variants, the metaanalyses were conducted comparing $7 R$ versus others, $4 R$ allele versus others and $2 R$ alleles versus others. Based on different pharmacological characteristics ${ }^{22,31}$, we divided these repeat alleles also into "short" (two to four) and "long" (five to eight) ${ }^{41-43}$ and conducted the metaanalyses considering "long" allele versus others.

\section{DRD4 polymorphisms in adults with ADHD}

Search strategy and selection criteria, inclusion and exclusion criteria, and statistical analyses were conducted as above, except for the term "adults" instead of "children OR child". During the research, we identified the most recent meta-analysis ${ }^{44}$, and we reported their findings, because no additional studies have been performed.

\section{Focus on DRD4 48 bp VNTRs polymorphism: functional differences}

We cross checked the references of the latest review describing the different studies on the functional biological effect of the $48 \mathrm{bp}$ VNTR polymorphism ${ }^{45}$ to find any publications possibly missed in our electronic search and did an updated search through to December 2018. We performed meta-analyses for $2 R$ allele versus $4 R, 2 R$ versus $7 R$ and $4 R$ versus $7 R$. Statistical analyses were conducted as above.

\section{Bioinformatics in silico analyses}

From 1000 Genome database in which the five SNPs found negative in last GWAS $^{15}$ (rs752306, rs7124601, rs146876215, rs1870723, rs7482904) are included, we built a population specific-linkage disequilibrium (LD) block by using Haploview software.

With the aim to further investigate the involvement of DRD4 on ADHD aetiology we performed a Transcription Wide Association Study (TWAS) considering the last available summary statistics for ADHD in the PGC portal (https://www.med.unc.edu/pgc/). TWAS is a gene association method estimating whether a different gene expression regulation (e.g., up or downregulation) could be expected 
for the analysed phenotype based on GWAS associations. This can be done through the imputation of the genetic component of gene expression using tissue-specific ciseQTL models ${ }^{46}$. In our analysis, we considered cis-eQTL models (http://predictdb.org/) trained on the GenotypeTissue Expression database, i.e., GTEx (https://gtexportal. org/home/) and we specifically focus on brain tissues.

\section{Results}

\section{Meta-analysis}

DRD4 polymorphisms in children with ADHD

48 bp VNTR polymorphism The PRISMA flow chart is in Supplementary Fig. S1. After screening 154 records, we selected 77 studies meeting our eligibility criteria: 43 studies case-control (CC), 21 family-based studies (TDT, transmission disequilibrium test) and 13 (combined case-control and transmission disequilibrium test approaches). Results in relation to different populations (Asian, European-Caucasian, Middle Eastern and South American) are reported in Table 1.

We structured this paragraph reporting the results in relation to (a) the comparisons using as dependent variable the allele comparison (allele $2 \mathrm{R}$ versus others; allele $4 R$ versus others; allele $7 R$ versus others; long allele versus others); (b) merged data between the two genetic approaches: CC and TDT studies for alleles 2R, 4R, 7R; (c) publication bias and (d) Newcastle-Ottawa Scale.

Allele $2 R$ versus others The results are showed in Supplementary Fig. S2 and summarized in Table 2.

In Asian populations: (a) CC: Random model $Z=0.27$, $P=0.79$, in presence of heterogeneity in effect size across the studies: $P=0.005, I^{2}=60 \%$; (b) TDT: Random model $Z=0.04, P=0.96$, in absence of heterogeneity in effect size across the studies: $P=0.56, I^{2}=0 \%$.

In European-Caucasian populations: (a) CC: Random model $Z=0.56, P=0.57$, without heterogeneity in effect size across the studies: $P=0.06, I^{2}=39 \%$; (b) TDT: Random model $Z=1.40, P=0.16$, without heterogeneity in effect size across the studies: $P=0.47, I^{2}=0 \%$.

In Middle Eastern populations: (a) CC: Random model $Z=0.79, P=0.43$, with heterogeneity in effect size across the studies: $P<0.0001, I^{2}=84 \%$; (b) TDT: Random model $Z=0.23, P=0.82$.

In South American populations: (a) CC: Random model $Z=0.61, P=0.54$, without heterogeneity in effect size across the studies: $P=0.12, I^{2}=49 \%$; (b) TDT: Random model $Z=0.82, P=0.41$.

Allele $4 R$ versus others The results are showed in Supplementary Fig. S3 and summarized in Table 2.

In Asian populations: (a) CC: Random model $Z=0.04$, $P=0.97$, without heterogeneity in effect size across the studies $P=0.11, I^{2}=36 \%$; (b) TDT: Random model
$Z=1.78, P=0.07$, with heterogeneity in effect size across the studies $P<0.00001, I^{2}=90 \%$.

In European-Caucasian populations: (a) CC: Random model $Z=3.31, P=0.0009, \mathrm{~d}=0.79$ 95\%CI: 0.69-0.91, with slightly heterogeneity in effect size across the studies $P=0.02, I^{2}=48 \%$; (b) TDT: Random model $Z=1.08$, $P=0.28$, with slightly heterogeneity in effect size across the studies $P=0.01, I^{2}=55 \%$.

In Middle Eastern populations: (a) CC: Random model $Z=0.31, P=0.76$, with heterogeneity in effect size across the studies $P<0.00001, I^{2}=87 \%$; (b) TDT: Random model $Z=0.72, P=0.47$.

In South American populations: (a) CC Random model $Z=1.66, P=0.10$, with no heterogeneity in effect size across the studies $P=0.08, I^{2}=50 \%$, (b) TDT: Random model $Z=0.00, P=1.00$.

Allele $7 R$ versus others The results are showed in Supplementary Fig. S4 and summarized in Table 2.

In Asian populations: (a) CC: Random model $Z=0.46$, $P=0.65$, without heterogeneity in effect size across the studies $P=0.77, I^{2}=0 \%$; (b) TDT: Random model $Z=$ $0.35, P=0.72$, without heterogeneity in effect size across the studies $P=0.60, I^{2}=0 \%$.

In European-Caucasian populations: (a) CC: Random model $Z=2.77, P=0.006, d=1.2595 \% \mathrm{CI}$ : $1.07-1.45$, with heterogeneity in effect size across the studies $P<$ $0.00001, I^{2}=75 \%$; (b) TDT Random model $Z=5.09, P<$ $0.00001, d=1.40$ 95\%CI: $1.23-1.59$ in absence of heterogeneity in effect size across the studies $P=0.25, I^{2}=16 \%$. In Middle Eastern populations: (a) CC: Random model $Z=3.13, P=0.002, d=0.6195 \% \mathrm{CI}$ : $0.45-0.83$ in absence of heterogeneity in effect size across the studies $P=0.50$, $I^{2}=0 \%$; (b) TDT: Random model $Z=0.63, P=0.53$, in absence of heterogeneity in effect size across the studies $P=0.11, I^{2}=61 \%$.

In South American populations: (a) CC: Random model $Z=1.59, P=0.11$, with a trend in heterogeneity in effect size across the studies $P=0.03, I^{2}=57 \%$; (b) TDT: Random model $Z=0.10, P=0.92$, in absence of heterogeneity in effect size across the studies $P=0.65$, $I^{2}=0 \%$.

Long allele versus others The results are showed in Supplementary Fig. S5 and summarized in Table 2.

In Asian populations: (a) CC: Random model $Z=1.01$, $P=0.31$, in absence of heterogeneity in effect size across the studies $P=0.43, I^{2}=1 \%$, (b) TDT: Random model $Z=0.94, P=0.35$, in absence of heterogeneity in effect size across the studies $P=0.19, I^{2}=35 \%$.

In European populations: (a) CC: Random model $Z=$ 4.04, $P<0.0001, d=1.41$ 95\%CI: $1.19-1.67$, in presence of heterogeneity in effect size across the studies $P=0.005$, $I^{2}=54 \%$, (b) TDT: Random model $Z=2.49, P=0.01$, 


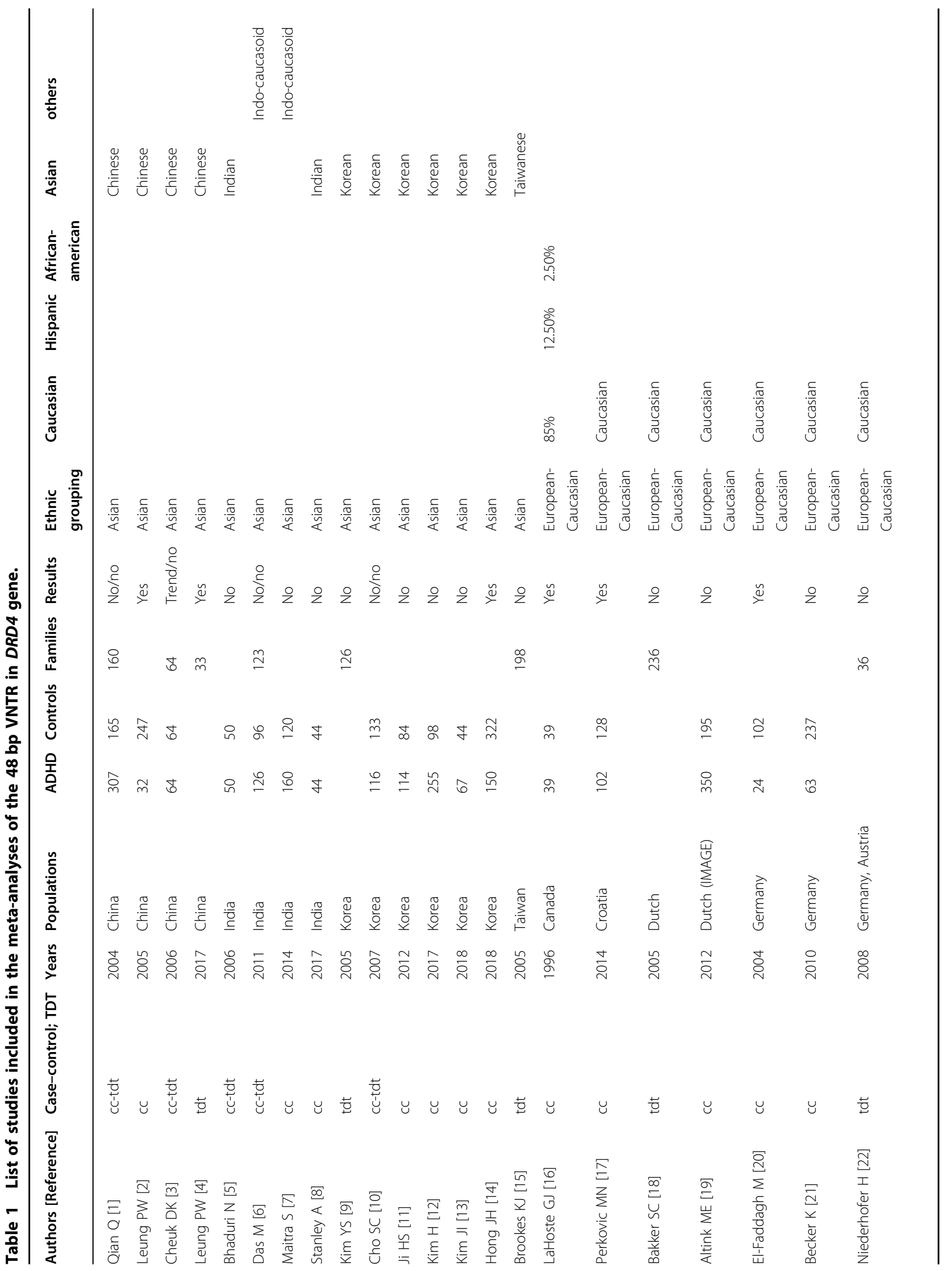




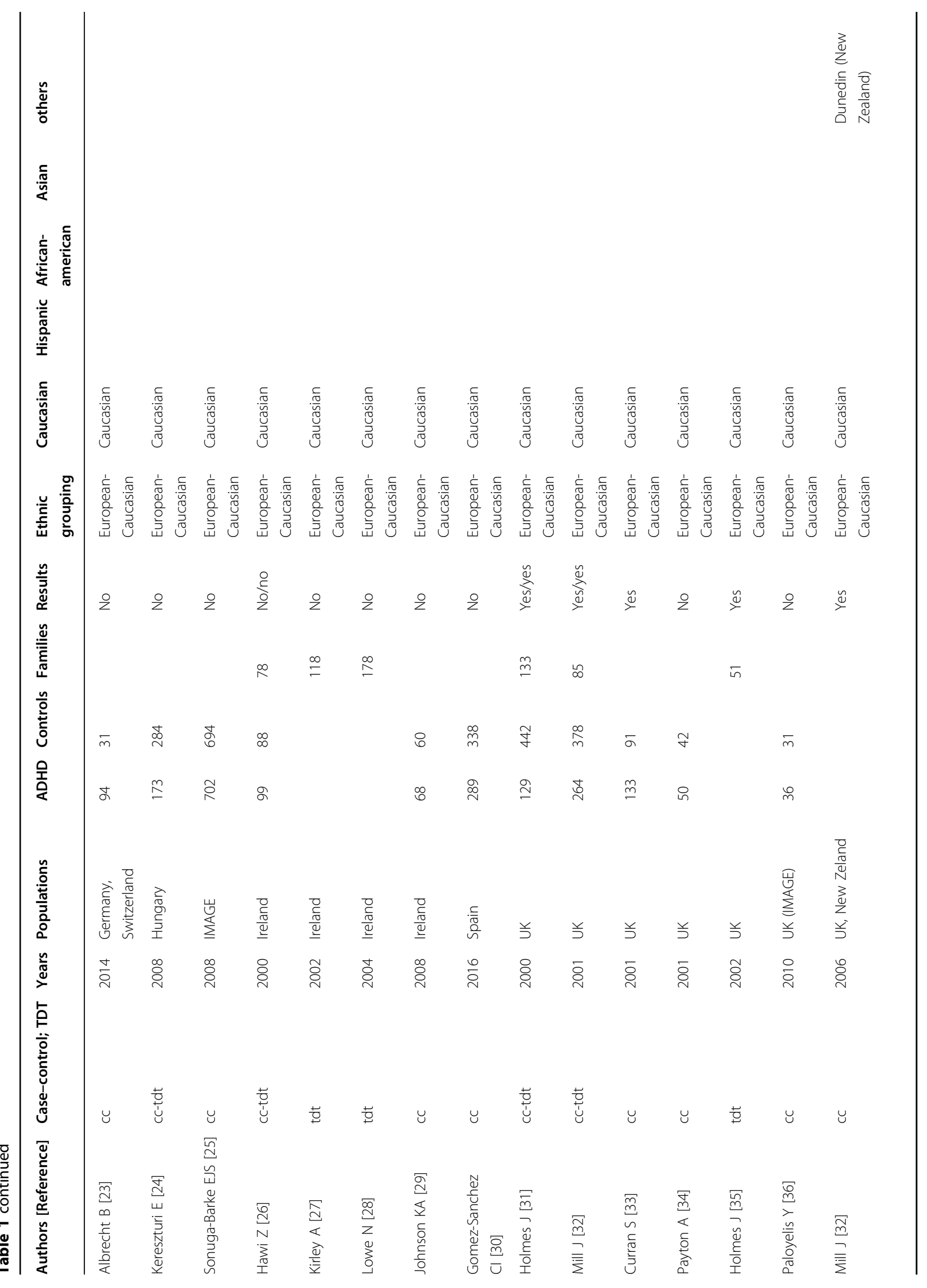




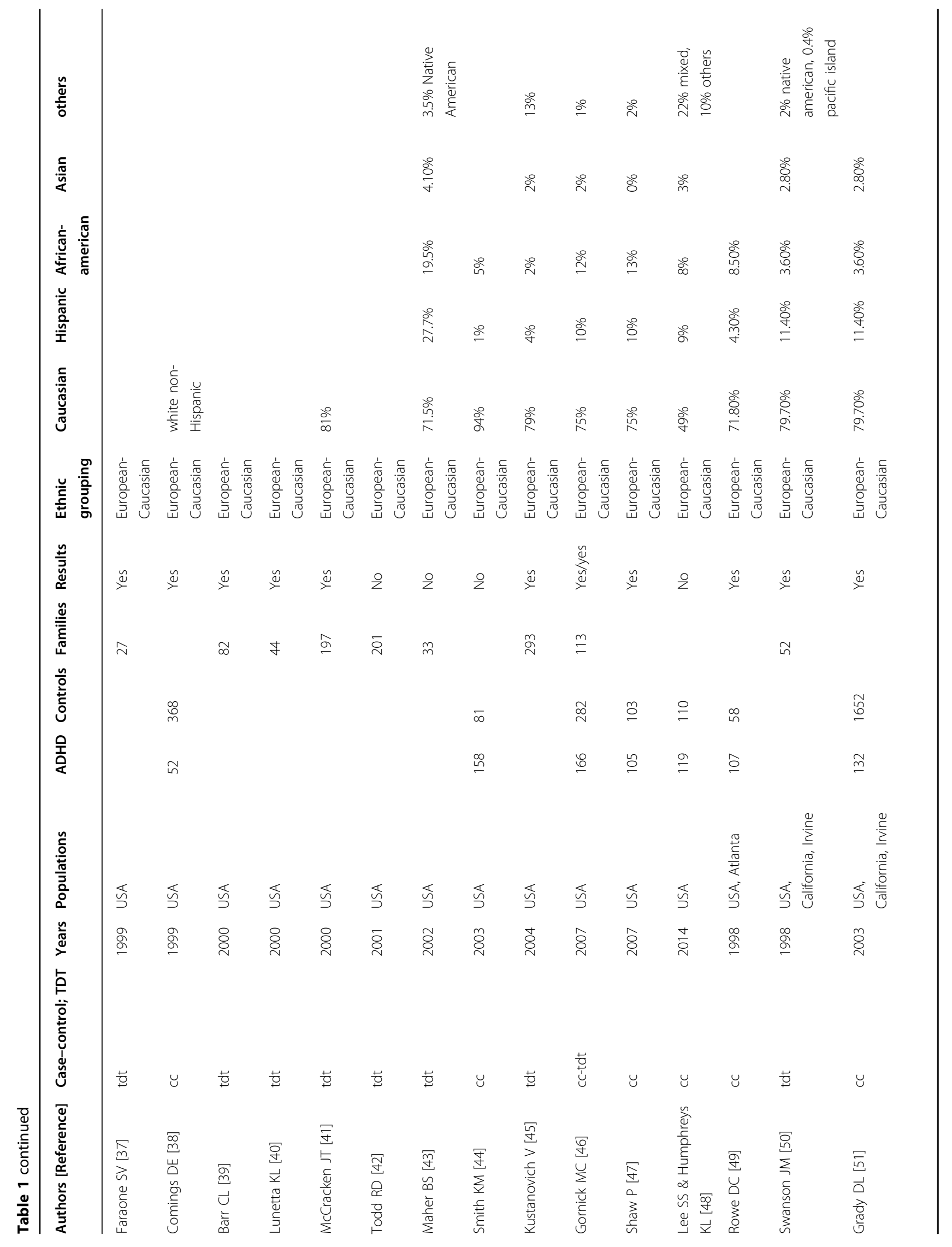




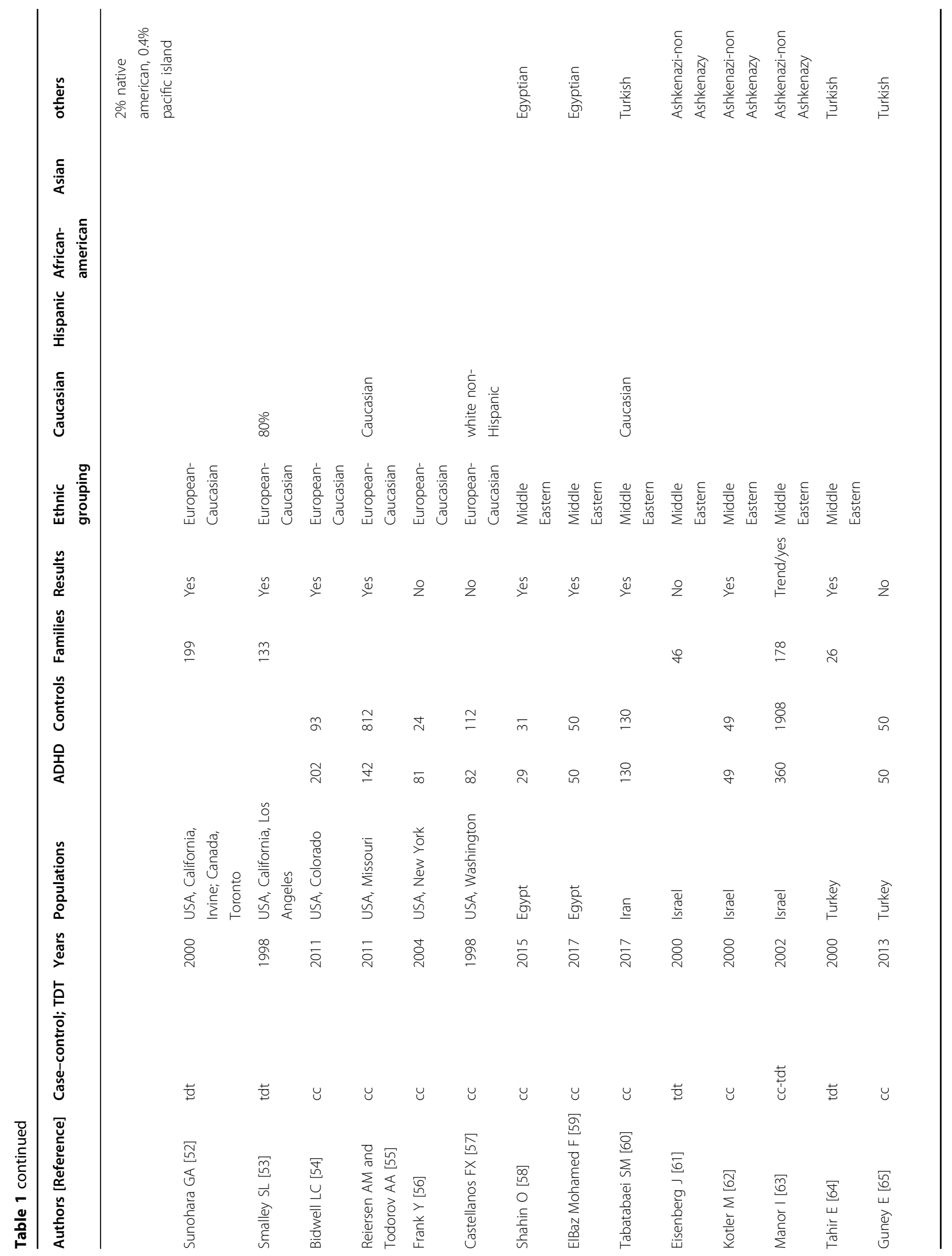




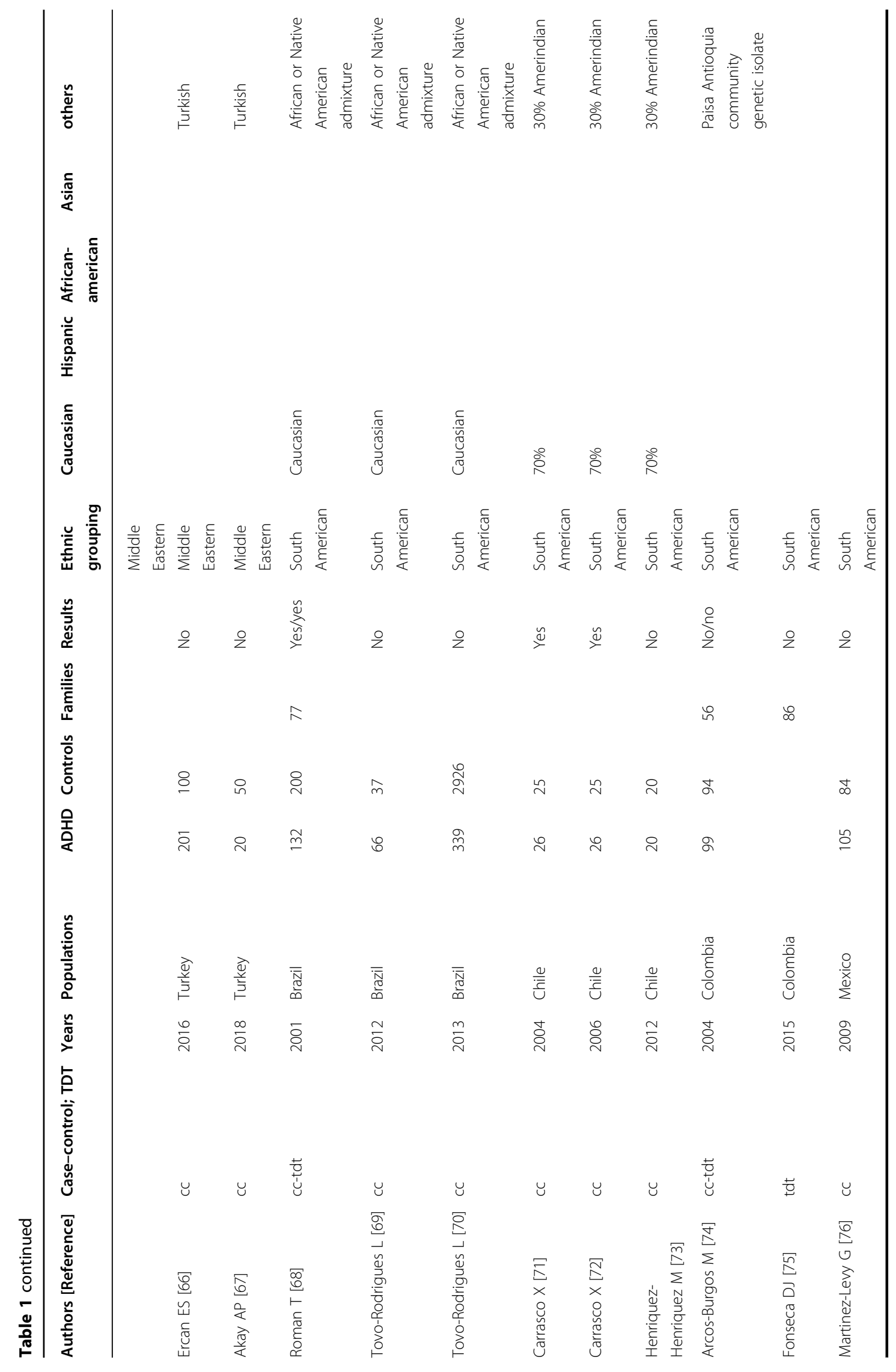




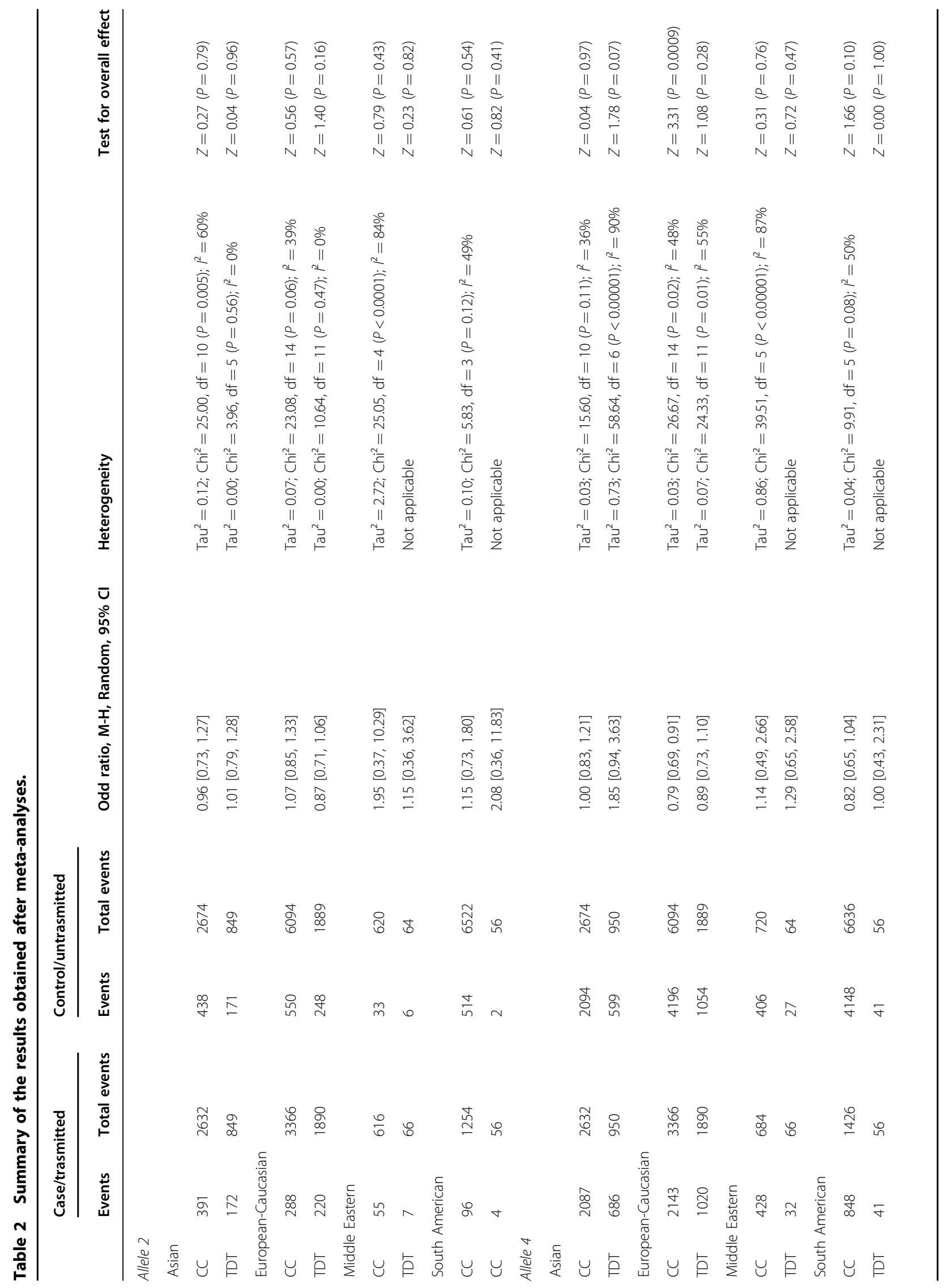




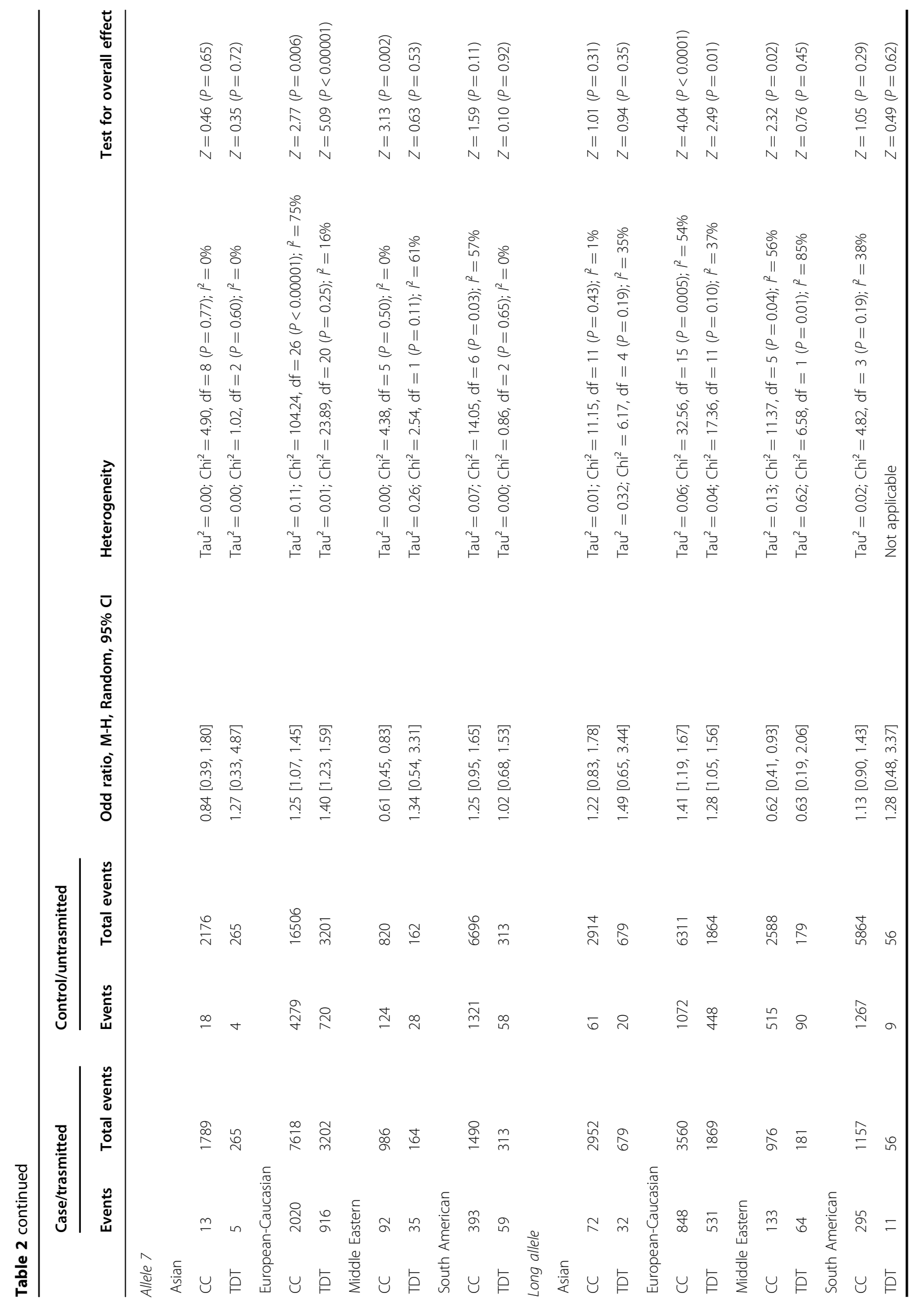


$d=1.2895 \%$ CI: $1.05-1.56$, in absence of heterogeneity in effect size across the studies $P=0.10, I^{2}=37 \%$.

In Middle Eastern populations: (a) CC: Random model $Z=2.32, P=0.02, d=0.62$ 95\%CI: $0.41-0.93$, with a trend of heterogeneity in effect size across the studies $P=0.04, I^{2}=56 \%$, (b) TDT: Random model $Z=0.76$, $P=0.45$, with heterogeneity in effect size across the studies $P=0.01, I^{2}=85 \%$.

In South American populations: (a) CC: Random model $Z=1.05, P=0.29$, in absence of heterogeneity in effect size across the studies $P=0.19, I^{2}=38 \%$, (b) TDT: Random model $Z=0.49, P=0.62$.

Merged data between the two approaches $C C$ and TDT for alleles $\mathbf{2 R}, \mathbf{4 R}, \mathbf{7 R}$ Table 3 shows the merged data from the CC and TDT studies.

The association with ADHD susceptibility was confirmed for allele $4 \mathrm{R}$ in European-Caucasian populations (Random model $Z=3.08, P=0.002, d=0.8395 \% \mathrm{CI}$ : 0.74-0.94, in presence of heterogeneity in effect size across the studies $P=0.0009, I^{2}=52 \%$ ). The statistical power increased when we combined the EuropeanCaucasian with South American populations (Random model $Z=3.58, P=0.0003, d=0.83$ 95\%CI: $0.75-0.92$ in presence of heterogeneity in effect size across the studies $P=0.0008, I^{2}=49 \%$ ). Allele 7R was found associated in the European-Caucasian populations (Random model $Z=4.70, \quad P<0.00001, d=1.3195 \% \mathrm{CI}: 1.17-1.47$, in presence of heterogeneity in effect size across the studies $\left.P<0.00001, I^{2}=66 \%\right)$.

Concerning the results for the "long" allele, we found associations with ADHD susceptibility in EuropeanCaucasian populations (Random model $Z=4.78, P<$ $0.00001, d=1.3695 \% \mathrm{CI}: 1.20-1.55$, in presence of heterogeneity in effect size across the studies $P=0.003$, $I^{2}=47 \%$ ), but with a protective effect in Middle Eastern population (Random model $Z=2.61, P=0.009, d=0.61$ 95\%CI: $0.42-0.88$, in presence of heterogeneity in effect size across the studies $P=0.009, I^{2}=63 \%$ ).

Publication bias The results of Egger's test for publication bias are reported in Supplementary Table S1. Publication bias was found for studies of the $7 R$ allele, mainly in the European-Caucasian populations $(P=$ 0.018), with higher values when the $C C$ and TDT findings were combined $(P=0.0004)$. Of note, we observed that, when we eliminated from the analyses the manuscripts from Sonuga-Barke and colleagues ${ }^{47}$ $(P=0.02)$ along with Altink and colleagues ${ }^{48}(P=0.08)$, the values are less significant and the $P$ value for the total sample was 0.83 .

Analyses of the "long" and 4R alleles showed no publication bias.
Newcastle-Ottawa Scale In Supplementary Table S2, we reported the results of the Newcastle-Ottawa Scale for this polymorphism.

SNPs Besides the VNTR, several SNPs were investigated. Our research did not add any other studies reported in the last meta-analysis by $\mathrm{Wu}$ and colleagues ${ }^{36}$. Thus, the results did not change for the $120 \mathrm{bp}$ duplication (rs4646984); -521 $(\mathrm{C} / \mathrm{T})$ (rs1800955); -616 (C/G) (rs747302), $12 \mathrm{bp}$ (rs46 46983); -615 (A/G) (rs936462); -376 (C/T) (rs916455), that did not show significant results.

For other SNPs: rs7395429, rs3758653, rs11246228, rs752306 ${ }^{49-51}$; rs4646984 ${ }^{52}$; rs916457 $7^{53}$; rs936465 ${ }^{54}$, nometa-analyses can be performed, because very few studies were available (minimum three studies), considering that $\mathrm{Yu}$ and colleagues $^{49,50}$ and Chang and colleagues ${ }^{51}$ studied the same population.

\section{DRD4 polymorphisms in MPH pharmacogenetic studies in children with $A D H D$}

Regarding to the research on the MPH pharmacogenetic studies, we ascertained that no other new studies were published on this topic as compared with the last meta-analysis by Myer and colleagues ${ }^{38}$ on 48 bp VNTR. Thus, we reported their results and their analyses. In particular, the homozygous $4 \mathrm{R}$ genotype demonstrated an association with improved MPH response, when compared with other genotypes (OR: $1.66,95 \%$ CI: 1.16-2.37, $P=0.005)$, whereas the meta-analysis of the $7 \mathrm{R}$ repeat allele versus others showed a trend with an $\mathrm{OR}=0.68$ (95\%CI: $0.47-1.00, P=0.05)^{38}$.

\section{DRD4 polymorphisms in adults with ADHD}

From the last meta-analysis ${ }^{44}$, no other studies on the topic were available to add to the analyses. Concerning 48 bp VNTR, no association was observed. Contrasting results have been reported for the $120 \mathrm{bp}$ duplication (rs4646984) and negative results for rs3758653, and rs936465. In relation to those retrieved in the most recent meta-analyses ${ }^{7,44}$, no other additional studies were found.

\section{DRD4 polymorphisms in MPH pharmacogenetic studies in adults with $A D H D$}

Concerning 48 bp VNTR, two studies were available with negative results and one study on $120 \mathrm{bp}$ duplication ${ }^{44}$.

\section{Focus on 48 bp VNTR in DRD4 gene: functional differences}

The last review by Pappa and colleagues ${ }^{45}$, that resumed the studies on the potential biological differences among DRD4 VNTR variants, was updated and, because no other new studies were conducted since 2014 to date, we conducted meta-analysis on the papers reported in Pappa and 


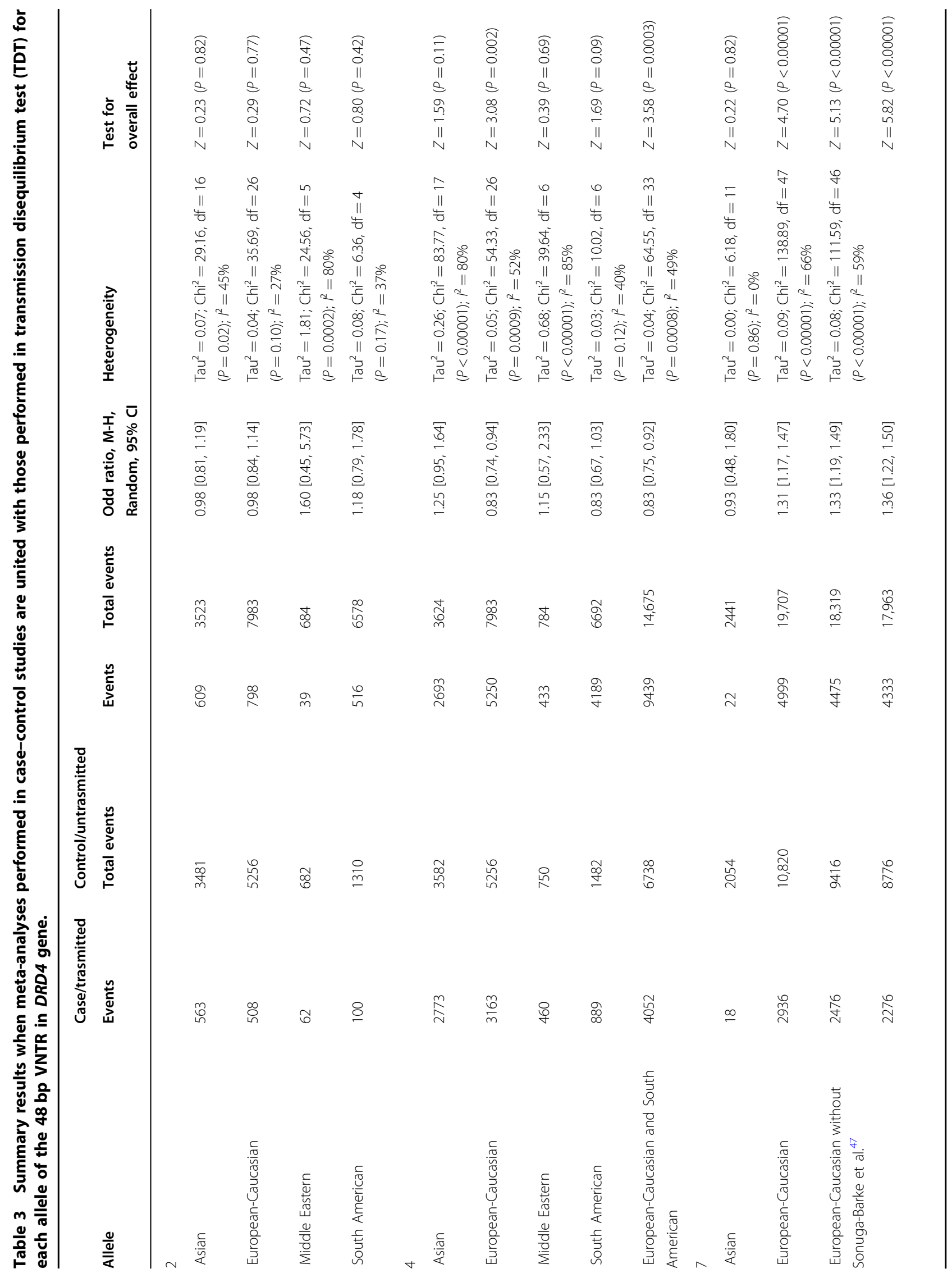




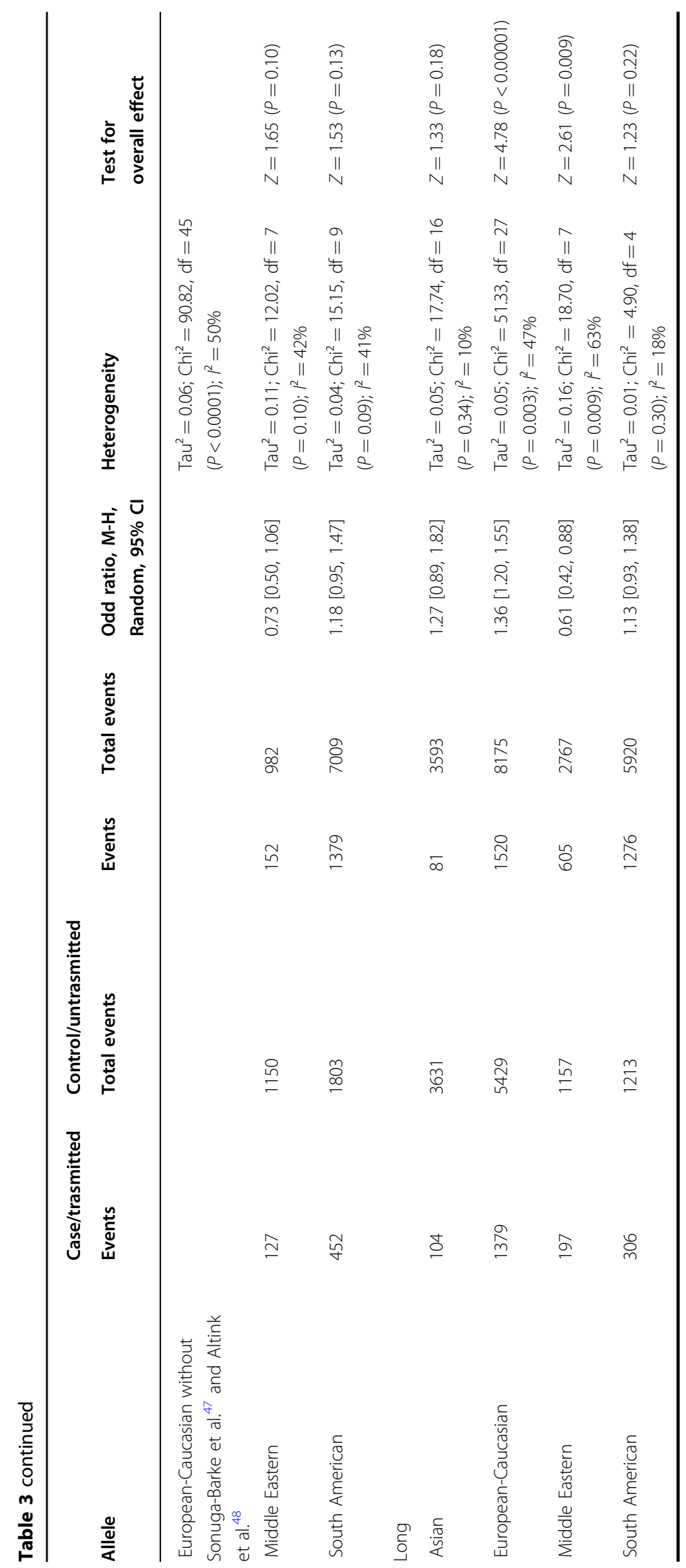


colleagues $^{45}$. The studies are divided according to in vitro, in vivo and in silico methodologies. There were enough studies (minimum three studies) to perform metaanalyses only for in vitro studies and they were divided according to technologies used: $\left[{ }^{3} \mathrm{H}\right]$ spiperone binding RIA; $\left[{ }^{3} \mathrm{H}\right]$ spiperone $\mathrm{Ca}^{2+}$ channel flux assay; $\left[{ }^{35} \mathrm{~S}\right] \mathrm{GTP}$ S agonist stimulated binding assay; $\mathrm{BRET}_{50}$ assay; luciferase reporter assay; western analysis; transient transfection. In Table 4, we reported these studies along with the techniques, functional response, the cell cultures used and the agonists. In Supplementary Figs. S6, S7, S8, the metaanalyses report the association of the functionality of allele $2 \mathrm{R}$ versus $4 \mathrm{R}$ (Random model $Z=4.52 ; P<0.00001$, $d=0.86$ 95\%CI: $0.48-1.23$ ); allele $2 \mathrm{R}$ versus 7R (Random model $Z=4.54 ; P<0.00001, d=1.07$ 95\%CI: 0.61-1.54) and allele 4R versus 7R (Random model $Z=4.81 ; P<$ $0.00001, d=1.2095 \%$ CI: $0.71-1.69$ ), respectively. These results showed evidence of decreased functionality of the $7 R$ compared with the $2 R$ and the $4 R$.

\section{Bioinformatics in silico analysis}

Using the 1000 Genomes Database, we built DRD4 gene LD blocks for different populations (African, American, East Asian, European and South Asian). We found that the 48 bp VNTR was not tagged by any of the GWAS SNPs used by Demontis and colleagues ${ }^{15}$ (Supplementary Fig. S9).

According to the brain tissues filter, the analysis showed a nominally significant association $(P<0.05)$ with $D R D 4$ due to a downregulation of gene expression in a specific brain area, which is the Putamen region included in Basal Ganglia $(Z$-score $=-3.02, P=0.00252)$.

\section{Discussion}

\section{Short summary of the major findings}

DRD4 48 bp VNTR appears to modulate the ADHD phenotype and MPH response across the lifespan, with differential associations depending on age and populations. This polymorphism has a significant impact on the pathophysiology, much more significant than the common SNPs variants.

Table 4 Summary of in vitro studies assessing functional differences among DRD4 VNTRs 48 bp.

\begin{tabular}{|c|c|c|c|c|c|c|}
\hline Authors & References & Years & Technique & Functional response & Cells & Agonist \\
\hline Asghari V et al. & {$[1]$} & 1994 & {$\left[{ }^{3} \mathrm{H}\right]$ spiperone binding RIA } & Non-specific & $\cos -7$ & Dopamine \\
\hline Asghari V et al. & {$[2]$} & 1995 & {$\left[{ }^{3} \mathrm{H}\right]$ spiperone binding RIA } & CAMP inhibition & $\mathrm{CHO}-\mathrm{K} 1$ & Dopamine \\
\hline Sanyal S \& Van Tol HH & [3] & 1997 & {$\left[{ }^{3} \mathrm{H}\right]$ spiperone binding RIA } & CAMP inhibition & $\mathrm{GH} 4 \mathrm{C} 1$ & Dopamine \\
\hline Oldenhof J et al. & [4] & 1998 & {$\left[{ }^{3} \mathrm{H}\right]$ spiperone binding RIA } & CAMP inhibition & $\mathrm{CHO}-\mathrm{K} 1$ & Dopamine \\
\hline Jovanovic $V$ et al. & {$[5]$} & 1999 & {$\left[{ }^{3} \mathrm{H}\right]$ spiperone binding RIA } & CAMP inhibition & $\mathrm{CHO}-\mathrm{K} 1$ & Dopamine \\
\hline Watts VJ et al. & {$[6]$} & 1999 & {$\left[{ }^{3} \mathrm{H}\right]$ spiperone binding RIA } & CAMP inhibition & HEK 293 & Dopamine \\
\hline Kazmi MA et al. & {$[7]$} & 2000 & $\begin{array}{l}{\left[{ }^{3} \mathrm{H}\right] \text { spiperone } \mathrm{Ca}^{2+} \text { channel }} \\
\text { flux assay }\end{array}$ & $\mathrm{Ca}^{2+}$ channel current inhibition & HEK 293T & Quinpirole \\
\hline Gilliland SL et al. & {$[8]$} & 2000 & $\begin{array}{l}{\left[{ }^{35} \text { S]GTPYS agonist stimulated }\right.} \\
\text { binding assay }\end{array}$ & $G_{i}$ protein & $\mathrm{CHO}-\mathrm{K} 1$ & Quinpirole \\
\hline Czermak C et al. & [9] & 2006 & $\begin{array}{l}{\left[{ }^{35} \mathrm{~S}\right] \mathrm{GTP} Y S \text { agonist stimulated }} \\
\text { binding assay }\end{array}$ & $\mathrm{G}_{i}$ protein & $\mathrm{CHO}-\mathrm{K} 1$ & Dopamine \\
\hline Van Craenenbroeck K et al. & {$[10]$} & 2011 & $\begin{array}{l}{\left[{ }^{35} \text { S]GTPYS agonist stimulated }\right.} \\
\text { binding assay }\end{array}$ & Non-specific & HEK 293T & Quinpirole \\
\hline Borroto-Escuela DO et al. & {$[11]$} & 2011 & BRET $_{50}$ assay & Receptors ratio & HEK 293T & $\mathrm{nr}$ \\
\hline Van Craenenbroeck K et al. & {$[10]$} & 2011 & BRET $_{50}$ assay & Non-specific & HEK 293T & Quinpirole \\
\hline Sanchez-Soto M et al. & {$[12]$} & 2016 & BRET $_{50}$ assay & CAMP inhibition & HEK 293T & Dopamine \\
\hline Sanchez-Soto M et al. & {$[13]$} & 2018 & $\mathrm{BRET}_{50}$ assay & $\mathrm{G}_{i}$ protein & HEK 293T & Dopamine \\
\hline Sanyal S \& Van Tol HH & [3] & 1997 & Luciferase reporter assay & CAMP inhibition & $\mathrm{GH} 4 \mathrm{C} 1$ & Quinpirole \\
\hline Schoots O \& Van Tol HH & {$[14]$} & 2003 & Luciferase reporter assay & Expression & $\mathrm{GH} 4 \mathrm{C} 1$ & $\mathrm{nr}$ \\
\hline Van Craenenbroeck K et al. & {$[15]$} & 2005 & Western analysis & Expression & $\mathrm{CHO}-\mathrm{K} 1$ & Quinpirole \\
\hline Gonzalez S et al. & {$[16]$} & 2012 & Transient transfection & $\begin{array}{l}\text { MAPK activation (ERK 1/2 } \\
\text { phosphorylation) }\end{array}$ & HEK 293T & $\mathrm{RO}-10-5824$ \\
\hline
\end{tabular}




\section{Findings in relation to the literature}

In our prior review ${ }^{32}$, we showed that the $7 \mathrm{R}$ allele, in childhood, has been associated with specific neuropsychological/neurophysiological tasks, brain structure and altered expression levels of $D R D 4$. We also found that the $7 R$ allele seems to moderate the effects of maternal smoking during pregnancy, season of birth, and parenting on externalizing behaviour in ADHD. The present study provides further evidence, with more updated meta-analyses, for the 7R/"long" allele as a strong ADHD susceptibility risk factor in European-Caucasian populations and that this allele leads to reduced biological functionality compared with the $2 \mathrm{R}$ and $4 \mathrm{R}$ alleles, modulating the receptor's signal transduction properties and altering intracellular cAMP level ${ }^{31}$. In other words, 7R allele has a reduced potency for coupling dopamine receptors to adenylate cyclase $^{31}$, and consequently a decreased dopamine sensitivity. More importantly, a further recent evidence $^{55}$ explores whether candidate genes are associated with multiple disorders via pleiotropic mechanisms, and/ or if other genes are specific to susceptibility for individual psychiatric disorders. Using a meta-analytic approach, the authors found that the 7R allele of $D R D 4$ was specifically implicated in ADHD and no with any other psychiatric diseases, validating our data both as regards the $7 \mathrm{R}$ allele as a major risk susceptibility factor for ADHD and as regards its specificity for ADHD. Of note, it results also specifically associated to childhood ADHD, and not in adult $\mathrm{ADHD}^{7,44}$. On the other hand, the $4 \mathrm{R} /$ "short" allele was a protective population-specific (European-Caucasian and South American) factor in children with ADHD, whereas our previous data ${ }^{44}$ supported no association in ADHD adulthood in general population.

As associations were observed also for the $S L C 6 A 3$ gene $^{7,55}$ where allelic variants showed differential effects in children and adults with ADHD, these findings suggest that DRD4 and SLC6A3 are among those genes that account for developmental variations with differential effects across the lifespan.

From the last SNPs/GWAS meta-analysis ${ }^{15}$, five SNPs in DRD4 were not significant according the GWAS cut-off significance $\left(10^{-8}\right)$. In this work, we show that those findings do not contradict our conclusions on the role of DRD4 in ADHD, because none of the SNPs assayed in that study ${ }^{15}$ are in LD with the $48 \mathrm{bp}$ VNTR. Thus, the role played by the DRD4 in ADHD susceptibility is determined predominantly by the $48 \mathrm{bp}$ VNTR variants.

The population-specific allelic heterogeneity we found is consistent with prior reports that the DRD4 VNTR displays a high degree of variability across populations worldwide, e.g. $48 \%$ in native Americans, but only $0-2 \%$ in Asians. There is no commonly accepted explanation for this variability at the DRD4 locus. A recent review ${ }^{56}$ suggested that the common and probably ancestral allele has four repeats, originating 300,000 years ago, whereas the $7 \mathrm{R}$ allele is up to 10 times younger. The $7 \mathrm{R}$ allele may have arisen as a rare mutational event and then become a high frequency allele by positive selection at a time of the major expansion of human population (the upper Paleolithic). In this way, individuals with novelty-seeking personality traits may have driven the expansion of the $7 R$ variant, or it may have conferred a reproductive advantage in male-competitive societies. In the Americas, an increase in the $7 \mathrm{R}$ allele may have been due to a successive founder effect, and in China a decrease in the $7 R$ may have been due to selective reproduction of males without the $7 \mathrm{R}$ allele. At the same time, there appears to be selective forces working to balance the alleles in modern societies (balancing selection), and the prevalence of the $7 \mathrm{R}$ allele may now be at a stable level or near a fixation point ${ }^{56}$.

Polymorphisms within key monoaminergic genes have been associated with the response to stimulant medication, albeit through conflicting evidence. This is mechanistically intuitive as MPH modulates extracellular catecholamine levels through interaction with dopaminergic, adrenergic and serotonergic system components. MPH inhibits catecholamine reuptake and modulates dopamine and norepinephrine levels, by binding to and blocking dopamine and norepinephrine transporters, thereby increasing extracellular concentrations ${ }^{57}$.

The most recent pharmacogenetics meta-analysis on the DRD4 48 bp VNTR ${ }^{38}$ reported a significant association between MPH efficacy and the 4R allele. ADHD children with $4 \mathrm{R} / 4 \mathrm{R}$ genotypes showed a $66 \%$ increased chance for efficacious MPH response; compared with others, where the efficacy measure was defined by changes at Clinical Global Impression-Improvement (CGI-I) and Severity (CGI-S), and ADHD Rating Scale (ADHD-RS), whereas the $7 R$ allele versus others did not reach significant association, even though a trend towards to poor $\mathrm{MPH}$ response was observed ${ }^{38}$. Thus, these data are in line with the European susceptibility/protection role of $7 R^{\prime \prime l o n g " / 4 R ~ a l l e l e s, ~ r e s p e c t i v e l y . ~ T h i s ~ i s ~ a l s o ~ c o n s i s t e n t ~}$ with the evidence that, as already evidenced, the $4 R$ leads to higher receptor expression and increased sensitivity to dopamine, as compared with the $7 \mathrm{R}$ variant. $\mathrm{MPH}$ works by blocking the pre-synaptic dopamine transporter, thus increasing synaptic dopamine ${ }^{58}$. Since $7 \mathrm{R}$ shows weaker transduction effects, the response to an increased level of synaptic dopamine will be weak ${ }^{31}$. These results further implicate that the children with ADHD homozygotes for $4 \mathrm{R}$ alleles would require lower doses of MPH to achieve symptom improvement.

The identification of predictors of pharmacotherapy is needed and always in development, to further the clinical implementation of precision medicine. Of note, patients receiving precision treatment were found to be more 
medication adherent ${ }^{59}$. Only half of children with ADHD followed pharmacological treatment regimens consistently over the course of a 5-year prospective study, and many reported adverse effects, and also the perceived tolerability may also be an impediment to adherence to treatments. Myer and colleagues ${ }^{38}$ analysed DNA variants in different genes linked to the effectiveness of MPH treatment. Leveraging individual genetic variants within not only DRD4 but also in SLC6A2, COMT, ADRA2A and $S L C 6 A 3$ the authors presented a plausible multivariate to assess risk for poor MPH efficacy. It is possible that, as they suggest, a multivariate predictor would be sufficiently accurate for clinical use. Furthermore, collectively evaluating genetic variability among plausible biological markers for treatment success would eliminate trial-and-error treatment used today ${ }^{60}$.

\section{Limitations}

We found, in some cases, heterogeneity in effect size across studies, and a significant Egger's test for funnel plot asymmetry which indicates presence of publication bias. Differences in sample and methodological approaches, absence of quality control analyses other than tests of Hardy-Weinberg equilibrium, absence of quality of the genotyping conducted, no repeated genotyping consistency, no call rates, and studies conducted in a wide time lapse (1996-2018), are some reasons for the presence of heterogeneity. Moreover, even though we conducted the analyses taking into consideration different populations ${ }^{37}$, some studies are not based on pure populations: i.e., refs. ${ }^{61-74}$ are primarily EuropeanCaucasian (about 80\%), but the remaining percentage of the sample also contain other ethnic groups (Table 1). Furthermore, even the studies ${ }^{75-81}$ performed in South American populations contains for about 70\% Caucasian samples, the remaining percentage is related to African or Native American admixture, Amerindian or Paisa Antioquia community genetic isolate (Table 1 ).

Other important sources of heterogeneity are linked to how the genotypic classification of alleles was conducted in different studies. Some used $7 \mathrm{R}$ carriers vs. non-carriers, others: $(2-5)$ vs. $(6-11)$ repeat carriers; $(2-6)$ vs. (7-11) repeat carriers; $(22,24,44)$ vs. $(27,47,77)$ genotypes; $2-4$ vs $5-11 \mathrm{R}$ carriers (for a review, see Pappa and colleagues ${ }^{45}$ ). We defined "short" allele (to $2 \mathrm{R}$ from $4 \mathrm{R}$ ), and "long" allele (to $5 \mathrm{R}$ from $8 \mathrm{R}$ ), a choice also confirmed by our data because the results did not change, as compared with the $4 R$ and $7 R$ analyses, respectively.

Finally, a TDT study design results significantly less heterogeneous than a CC study. Thus, we suggest conducting the meta-analyses, taking in consideration study design (differently from the previous meta-analyses ${ }^{35,36}$ ).

Regarding the results from Egger's test, for the 7R case, we observed presence of publication bias in European populations with a CC model $(P=0.018)$, but the $P$ value becomes smaller $(P=0.0004)$ when $C$ model is merged with TDT study design. We observed that, when we eliminated from the analyses Sonuga-Barke and colleagues $^{47}$ along with Altink and colleagues ${ }^{48}$, the values are less significant and the $P$ value for the total sample was 0.83 . This could further mean the importance of studying this kind of polymorphism in samples where there are not mixed populations.

\section{Conclusions and future directions}

Our data strongly suggest that DRD 448 bp VNTR could influence the ADHD susceptibility as well as the MPH response across the lifespan, with differential associations depending on age and populations. Interestingly, as compared with the other common SNPs variants, this VNTR polymorphism shows a significant impact on the pathophysiology of ADHD.

The advent of the new and high-throughput technologies such as next generation sequencing are contributing to better elucidate the implication of the rare variants on the ADHD susceptibility: interestingly it has been observed an increased burden of rare variants inside the 7R allele of DRD4 both in ADHD children ${ }^{72}$, and in adults $^{75}$ that needed further investigation.

In the era of precision medicine, the identification of biomarkers associated to diagnosis and treatment represents a valid way to classify complex mental disorders such as ADHD and offers the opportunity to standardize and improve diagnostic assessment, provide insights into etiological mechanisms, and contribute to developing individualized therapies. Although biomarkers are successfully used in predicting diseases such as cancer, there is no lab test that is used clinically for the diagnosis of ADHD. While there are several pharmacological treatments for ADHD, the mechanisms of action of these agents are still unclear and no specific biological predictors of treatment response are available. We here want to strength the added value provided by the biomarker identification approach for ADHD, and even though future work is needed, we speculate that $7 R$ and $4 R$ alleles of the $48 \mathrm{bp}$ VNTR can contribute to improve the diagnostic picture with their specificity to childhood ADHD and to be a further actor in that possible multivariate predictor $^{38}$ to the MPH response that could be sufficiently accurate for clinical use.

\section{Acknowledgements}

This research was supported by grants from the Italian Ministry of Health (Ricerca Corrente). This project has received funding from the European Union's Horizon 2020 research and innovation program grant agreement No. 667302.

\section{Author details}

${ }^{1}$ Molecular Markers Laboratory, IRCCS Istituto Centro San Giovanni di Dio Fatebenefratelli, Brescia, Italy. ${ }^{2}$ Center for Innovation in Mental Health, 
Academic Unit of Psychology, University of Southampton, Southampton SO17 1BJ, UK. ${ }^{3}$ Clinical and Experimental Sciences (CNS and Psychiatry), Faculty of Medicine, University of Southampton, Southampton SO17 1BJ, UK. ${ }^{4}$ Solent NHS Trust, Southampton SO19 8BR, UK. ${ }^{5}$ New York University Child Study Center, New York, NY 10016, USA. 'Division of Psychiatry and Applied Psychology, School of Medicine, University of Nottingham, Nottingham NG72UH, UK ${ }^{7}$ Institute for Genomic Statistics and Bioinformatics, University Hospital Bonn, Bonn, Germany. ${ }^{8}$ Department of Psychiatry, University of Münster, Münster, Germany. ${ }^{9}$ Department of Psychiatry, Melbourne Medical School, The University of Melbourne, Melbourne, VIC, Australia. ${ }^{10}$ The Florey Institute of Neuroscience and Medical Health, The University of Melbourne, Parkville, VIC, Australia. ${ }^{11}$ Departments of Psychiatry and of Neuroscience and Physiology, SUNY Upstate Medical University, Syracuse 13210 NY, USA. ${ }^{12}$ K.G. Jebsen Centre for Research on Neuropsychiatric Disorders, University of Bergen, Bergen, Norway. ${ }^{13}$ Biological Psychiatry Unit, IRCCS Istituto Centro San Giovanni di Dio Fatebenefratelli, Brescia, Italy

\section{Conflict of interest}

Cristian Bonvicini Ph.D., Catia Scassellati, PhD, Carlo Maj and Bernhard T. Baune declare that they have no conflict of interest. Prof. Steve Faraone, in the past year, received income, potential income, travel expenses continuing education support and/or research support from Tris, Otsuka, Arbor, Ironshore, Shire, Akili Interactive Labs, VAYA, Ironshore, Sunovion, Supernus and Genomind. With his institution, he has US patent US20130217707 A1 for the use of sodiumhydrogen exchange inhibitors in the treatment of $\mathrm{ADHD}$. He also receives royalties from books published by Guilford Press: Straight Talk about Your Child's Mental Health, Oxford University Press: Schizophrenia: The Facts and Elsevier: ADHD: Non-Pharmacologic Interventions. He is the principal investigator of www.adhdinadults.com. Prof. Samuele Cortese: Dr Cortese reports receiving reimbursement for travel and accommodation expenses from the Association for Child and Adolescent Central Health (ACAMH), a nonprofit organization, in relation to lectures that he delivered for ACAMH and by Healthcare convention for educational activity on ADHD.

\section{Publisher's note}

Springer Nature remains neutral with regard to jurisdictional claims in published maps and institutional affiliations.

Supplementary Information accompanies this paper at (https://doi.org/ 10.1038/s41398-020-0755-4).

Received: 20 February 2019 Revised: 10 December 2019 Accepted: 20 December 2019

Published online: 19 February 2020

\section{References}

1. Faraone, S. V. et al. Attention-deficit/hyperactivity disorder. Nat. Rev. Dis. Prim. 1, 15020 (2015)

2. Polanczyk, G., de Lima, M. S., Horta, B. L., Biederman, J. \& Rohde, L. A. The worldwide prevalence of ADHD: a systematic review and metaregression analysis. Am. J. Psychiatry 164, 942-948 (2007).

3. Faraone, S. V., Biederman, J. \& Mick, E. The age-dependent decline of attention deficit hyperactivity disorder: a meta-analysis of follow-up studies. Psychol. Med. 36, 159-165 (2006)

4. Simon, V., Czobor, P., Balint, S., Meszaros, A. \& Bitter, I. Prevalence and correlates of adult attention-deficit hyperactivity disorder: meta-analysis. Br. J. Psychiatry 194, 204-211 (2009).

5. Haavik, J., Halmoy, A., Lundervold, A. J. \& Fasmer, O. B. Clinical assessment and diagnosis of adults with attention-deficit/hyperactivity disorder. Expert Rev. Neurother. 10, 1569-1580 (2010).

6. Hoogman, M. et al. Subcortical brain volume differences in participants with attention deficit hyperactivity disorder in children and adults: a cross-sectional mega-analysis. Lancet Psychiatry 4, 310-319 (2017).

7. Bonvicini, C., Faraone, S. V. \& Scassellati, C. Common and specific genes and peripheral biomarkers in children and adults with attention-deficit/hyperactivity disorder. World J. Biol. Psychiatry 19, 80-100 (2018).

8. Faraone, S. V. et al. Molecular genetics of attention-deficit/hyperactivity disorder. Biol. Psychiatry 57, 1313-1323 (2005).
9. Faraone, S. V. \& Larsson, H. Genetics of attention deficit hyperactivity disorder. Mol. Psychiatry 24, 562-575 (2019).

10. Boomsma, D. I. et al. Genetic epidemiology of attention deficit hyperactivity disorder (ADHD index) in adults. PLoS ONE 5, e10621 (2010).

11. Kan, K. J. et al. Genetic and environmental stability in attention problems across the lifespan: evidence from the Netherlands twin register. J. Am. Acad. Child Adolesc. Psychiatry 52, 12-25 (2013).

12. Larsson, $\mathrm{H}$. et al. Genetic and environmental influences on adult attention deficit hyperactivity disorder symptoms: a large Swedish population-based study of twins. Psychol. Med. 43, 197-207 (2013).

13. Biederman, J. et al. Predictors of persistence and remission of ADHD into adolescence: results from a 4-year prospective follow-up study. J. Am. Acad. Child Adolesc. Psychiatry 35, 343-351 (1996).

14. Faraone, S. V. et al. Attention-deficit/hyperactivity disorder in adults: an overview. Biol. Psychiatry 48, 9-20 (2000).

15. Demontis, D. et al. Discovery of the first genome-wide significant risk loci for attention deficit/hyperactivity disorder. Nat. Genet. 51, 63-75 (2019).

16. Kornfield, R. et al. Effects of FDA advisories on the pharmacologic treatment of ADHD, 2004-2008. Psychiatr. Serv. 64, 339-346 (2013).

17. Cortese, $\mathrm{S}$. et al. Comparative efficacy and tolerability of medications for attention-deficit hyperactivity disorder in children, adolescents, and adults: a systematic review and network meta-analysis. Lancet Psychiatry 5, 727-738 (2018).

18. Callier, S. et al. Evolution and cell biology of dopamine receptors in vertebrates. Biol. Cell. 95, 489-502 (2003).

19. Oldenhof, J. et al. SH3 binding domains in the dopamine D4 receptor. Biochemistry 37, 15726-15736 (1998).

20. Floresco, S. B. \& Tse, M. T. Dopaminergic regulation of inhibitory and excitatory transmission in the basolateral amygdala-prefrontal cortical pathway. J. Neurosci. 27, 2045-2057 (2007).

21. Noain, D. et al. Identification of brain neurons expressing the dopamine D4 receptor gene using BAC transgenic mice. Eur. J. Neurosci. 24, 2429-2438 (2006).

22. Benjamin, J. et al. Population and familial association between the D4 dopamine receptor gene and measures of Novelty Seeking. Nat. Genet. 12, 81-84 (1996).

23. Ebstein, R. P. et al. Dopamine D4 receptor (D4DR) exon III polymorphism associated with the human personality trait of Novelty Seeking. Nat. Genet. 12 78-80 (1996).

24. Faraone, S. V. et al. Dopamine D4 gene 7-repeat allele and attention deficit hyperactivity disorder. Am. J. Psychiatry 156, 768-770 (1999).

25. Rubinstein, M. et al. Mice lacking dopamine D4 receptors are supersensitive to ethanol, cocaine, and methamphetamine. Cell 90, 991-1001 (1997).

26. Chang, F. M., Kidd, J. R., Livak, K. J., Pakstis, A. J. \& Kidd, K. K. The world-wide distribution of allele frequencies at the human dopamine D4 receptor locus. Hum. Genet. 98, 91-101 (1996).

27. Bhaduri, N., Das, M., Das, A. B. \& Mukhopadhyay, K. Dopamine receptor D4 exon 3 variable number of tandem repeat polymorphism: distribution in eastern Indian population. Indian J. Hum. Genet. 13, 54-58 (2007).

28. Mansoor, A., Mazhar, K. \& Qamar, R. VNTR polymorphism of the DRD4 locus in different Pakistani ethnic groups. Genet Test. 12, 299-304 (2008).

29. Baydala, L., Sherman, J., Rasmussen, C., Wikman, E. \& Janzen, H. ADHD characteristics in Canadian Aboriginal children. J. Atten. Disord. 9, 642-647 (2006).

30. Borinskaia, S. A. et al. Analysis of the DRD4 gene polymorphism in populations of Russia and neighboring countries. Genetika 40, 835-840 (2004).

31. Asghari, V. et al. Modulation of intracellular cyclic AMP levels by different human dopamine D4 receptor variants. J. Neurochem. 65, 1157-1165 (1995).

32. Faraone, S. V., Bonvicini, C. \& Scassellati, C. Biomarkers in the diagnosis of ADHD-promising directions. Curr. Psychiatry Rep. 16, 014-0497-1 (2014).

33. Bonvicini, C., Maj, C. \& Scassellati, C. Genetics and pharmacogenetics of attention deficit hyperactivity disorder in childhood and adulthood. Personalised Psychiatry (ed. Bernhard T. Baune), Academic Press, 253-274 (2020).

34. Moher, D., Liberati, A., Tetzlaff, J. \& Altman, D. G. PRISMA Group. Preferred reporting items for systematic reviews and meta-analyses: the PRISMA statement. PLoS Med. 6, e1000097 (2009).

35. Gizer, I. R., Ficks, C. \& Waldman, I. D. Candidate gene studies of ADHD: a metaanalytic review. Hum. Genet. 126, 51-90 (2009).

36. Wu, J., Xiao, H., Sun, H., Zou, L. \& Zhu, L. Q. Role of dopamine receptors in ADHD: a systematic meta-analysis. Mol. Neurobiol. 45, 605-620 (2012).

37. Nikolaidis, A.\& Gray, J. R. ADHD and the DRD4 exon III 7-repeat polymorphism: an international meta-analysis. Soc. Cogn. Affect Neurosci. 5, 188-193 (2010). 
38. Myer, N. M., Boland, J. R. \& Faraone, S. V. Pharmacogenetics predictors of methylphenidate efficacy in childhood ADHD. Mol. Psychiatry 23, 1-8 (2018).

39. Wells, G. A. et al. The Newcastle-Ottawa scale (NOS) for assessing the quality of nonrandomized studies in meta-analyses. http://www.ohri.ca/programs/ clinical_epidemiology/oxford.asp.

40. Egger, M., Smith, G. D. \& Phillips, A. N. Meta-analysis: principles and procedures. BMJ 315, 1533-1537 (1997).

41. Bhaduri, N. et al. Association of dopamine D4 receptor (DRD4) polymorphisms with attention deficit hyperactivity disorder in Indian population. Am. J. Med. Genet. B Neuropsychiatr Genet. 141B, 61-66 (2006).

42. Comings, D. E. et al. Studies of the $48 \mathrm{bp}$ repeat polymorphism of the DRD4 gene in impulsive, compulsive, addictive behaviors: Tourette syndrome, ADHD, pathological gambling, and substance abuse. Am. J. Med. Genet. 88, 358-368 (1999).

43. Hawi, Z. et al. No association of the dopamine DRD4 receptor (DRD4) gene polymorphism with attention deficit hyperactivity disorder (ADHD) in the Irish population. Am. J. Med. Genet. 96, 268-272 (2000).

44. Bonvicini, C., Faraone, S. V. \& Scassellati, C. Attention-deficit hyperactivity disorder in adults: A systematic review and meta-analysis of genetic, pharmacogenetic and biochemical studies. Mol. Psychiatry 21, 872-884 (2016).

45. Pappa, I., Mileva-Seitz, V. R., Bakermans-Kranenburg, M. J., Tiemeier, H. \& van IJzendoorn, M. H. The magnificent seven: a quantitative review of dopamine receptor $\mathrm{d} 4 \mathrm{and}$ its association with child behavior. Neurosci. Biobehav. Rev. 57, 175-186 (2015).

46. Gamazon, E. R. et al. A gene-based association method for mapping traits using reference transcriptome data. Nat. Genet. 47, 1091-1098 (2015).

47. Sonuga-Barke, E. J. et al. Intelligence in DSM-IV combined type attentiondeficit/hyperactivity disorder is not predicted by either dopamine receptor/ transporter genes or other previously identified risk alleles for attention-deficit/ hyperactivity disorder. Am. J. Med. Genet. B Neuropsychiatr. Genet. 147, 316-319 (2008).

48. Altink, M. E. et al. The dopamine receptor D4 7-repeat allele influences neurocognitive functioning, but this effect is moderated by age and ADHD status: an exploratory study. World J. Biol. Psychiatry 13, 293-305 (2012).

49. Yu, C. J. et al. Increased risk of attention-deficit/hyperactivity disorder associated with exposure to organophosphate pesticide in Taiwanese children. Andrology 4, 695-705 (2016).

50. Yu, C. J. et al. Attention deficit/hyperactivity disorder and urinary nonylphenol levels: a case-control study in Taiwanese children. PLOS ONE 11, e0149558 (2016).

51. Chang, C. H. et al. The interactions among organophosphate pesticide exposure, oxidative stress, and genetic polymorphisms of dopamine receptor D4 increase the risk of attention deficit/hyperactivity disorder in children. Environ. Res. 160, 339-346 (2018).

52. Ghosh, P. et al. Catecholaminergic gene variants: contribution in ADHD and associated comorbid attributes in the eastern Indian probands. Biomed. Res. Int. 2013, 918410 (2013).

53. Brookes, K. J. et al. A common haplotype of the dopamine transporter gene associated with attention-deficit/hyperactivity disorder and interacting with maternal use of alcohol during pregnancy. Arch. Gen. Psychiatry 63, 74-81 (2006).

54. Ribases, M. et al. Candidate system analysis in ADHD: evaluation of nine genes involved in dopaminergic neurotransmission identifies association with DRD1. World J. Biol. Psychiatry 13, 281-292 (2012).

55. Gatt, J. M., Burton, K. L., Williams, L. M. \& Schofield, P. R. Specific and common genes implicated across major mental disorders: a review of meta-analysis studies. J. Psychiatr. Res. 60, 1-13 (2015).

56. Turic, D., Swanson, J. \& Sonuga-Barke, E. DRD4 and DAT1 in ADHD: functional neurobiology to pharmacogenetics. Pharmgenomics Pers. Med. 3, 61-78 (2010).

57. Faraone, S. V. The pharmacology of amphetamine and methylphenidate: relevance to the neurobiology of attention-deficit/hyperactivity disorder and other psychiatric comorbidities. Neurosci. Biobehav Rev. 87, 255-270 (2018).

58. Seeman, P. \& Madras, B. K. Anti-hyperactivity medication: methylphenidate and amphetamine. Mol. Psychiatry 3, 386-396 (1998).

59. Fagerness, J. et al. Pharmacogenetic-guided psychiatric intervention associated with increased adherence and cost savings. Am. J. Manag Care. 20, e146-e156 (2014).
60. Stein, M. A. \& McGough, J. J. The pharmacogenomic era: promise for personalizing attention deficit hyperactivity disorder therapy. Child Adolesc. Psychiatr. Clin. N. Am. 17, xi-xii (2008).

61. LaHoste, G. J. et al. Dopamine D4 receptor gene polymorphism is associated with attention deficit hyperactivity disorder. Mol. Psychiatry 1, 121-124 (1996).

62. Mill, J. et al. Prediction of heterogeneity in intelligence and adult prognosis by genetic polymorphisms in the dopamine system among children with attention-deficit/hyperactivity disorder: evidence from 2 birth cohorts. Arch. Gen. Psychiatry 63, 462-469 (2006)

63. McCracken, J. T. et al. Evidence for linkage of a tandem duplication polymorphism upstream of the dopamine D4 receptor gene (DRD4) with attention deficit hyperactivity disorder (ADHD). Mol. Psychiatry $\mathbf{5}$, 531-536 (2000).

64. Maher, B. S., Marazita, M. L., Ferrell, R. E. \& Vanyukov, M. M. Dopamine system genes and attention deficit hyperactivity disorder: a meta-analysis. Psychiatr. Genet. 12, 207-215 (2002).

65. Smith, K. M. et al. Association of the dopamine beta hydroxylase gene with attention deficit hyperactivity disorder: genetic analysis of the Milwaukee longitudinal study. Am. J. Med. Genet. B Neuropsychiatr. Genet. 119B, 77-85 (2003).

66. Kustanovich, $\mathrm{V}$. et al. Transmission disequilibrium testing of dopamine-related candidate gene polymorphisms in ADHD: confirmation of association of ADHD with DRD4 and DRD5. Mol. Psychiatry 9, 711-717 (2004).

67. Gornick, M. C. et al. Association of the dopamine receptor D4 (DRD4) gene 7repeat allele with children with attention-deficit/hyperactivity disorder (ADHD): an update. Am. J. Med. Genet. B Neuropsychiatr. Genet. 144B, 379-382 (2007).

68. Shaw, P. et al. Polymorphisms of the dopamine D4 receptor, clinical outcome, and cortical structure in attention-deficit/hyperactivity disorder. Arch. Gen. Psychiatry 64, 921-931 (2007).

69. Lee, S. S. \& Humphreys, K. L. Interactive association of dopamine receptor (DRD4) genotype and ADHD on alcohol expectancies in children. Exp. Clin. Psychopharmacol. 22, 100-109 (2014).

70. Rowe, D. C. et al. Dopamine DRD4 receptor polymorphism and attention deficit hyperactivity disorder. Mol. Psychiatry 3, 419-426 (1998).

71. Swanson, J. M. et al. Association of the dopamine receptor D4 (DRD4) gene with a refined phenotype of attention deficit hyperactivity disorder (ADHD): a family-based approach. Mol. Psychiatry 3, 38-41 (1998).

72. Grady, D. L. et al. High prevalence of rare dopamine receptor D4 alleles in children diagnosed with attention-deficit hyperactivity disorder. Mol. Psychiatry 8, 536-545 (2003).

73. Smalley, S. L. et al. Evidence that the dopamine D4 receptor is a susceptibility gene in attention deficit hyperactivity disorder. Mol. Psychiatry 3, 427-430 (1998).

74. Castellanos, F. X. et al. Lack of an association between a dopamine-4 receptor polymorphism and attention-deficit/hyperactivity disorder: genetic and brain morphometric analyses. Mol. Psychiatry 3, 431-434 (1998).

75. Tovo-Rodrigues, L. et al. Is there a role for rare variants in DRD4 gene in the susceptibility for ADHD? Searching for an effect of allelic heterogeneity. Mol. Psychiatry 17, 520-526 (2012).

76. Arcos-Burgos, M. et al. Pedigree disequilibrium test (PDT) replicates association and linkage between DRD4 and ADHD in multigenerational and extended pedigrees from a genetic isolate. Mol. Psychiatry 9, 252-259 (2004).

77. Carrasco, X. et al. Presence of DRD4/7R and DAT1/10R allele in Chilean family members with attention deficit hyperactivity disorder. Rev. Med. Chil. 132, 1047-1052 (2004)

78. Carrasco, X. et al. Genotypic interaction between DRD4 and DAT1 loci is a high risk factor for attention-deficit/hyperactivity disorder in Chilean families. Am. J. Med. Genet. B Neuropsychiatr. Genet. 141B, 51-54 (2006).

79. Henriquez-Henriquez, M. et al. Intratask Variability As a Correlate for DRD4 and SLC6A3 Variants: A Pilot Study in ADHD. J. Atten. Disord. 19, 987-996 (2015).

80. Tovo-Rodrigues, L. et al. DRD4 rare variants in Attention-Deficit/Hyperactivity Disorder (ADHD): further evidence from a birth cohort study. PLoS One $\mathbf{8}$, e85164 (2013).

81. Roman, T. et al. Attention-deficit hyperactivity disorder: a study of association with both the dopamine transporter gene and the dopamine D4 receptor gene. Am. J. Med. Genet. 105, 471-478 (2001). 